\title{
CRESCIMENTO DE MUDAS DE Bauhinia forficata Link. EM DIFERENTES TAMANHOS DE RECIPIENTES
}

\author{
Jeandson Silva Viana ${ }^{1}$, Edilma Pereira Gonçalves ${ }^{2}$, Leonaldo Alves de Andrade ${ }^{3}$, \\ Lamartine Soares Bezerra de Oliveira ${ }^{4}$, Edna de Oliveira Silva ${ }^{5}$ \\ ${ }^{1}$ Eng. Agrônomo, Dr., Unidade Acadêmica de Serra Talhada, UFRPE, Serra Talhada, PE, Brasil - jeandson@uast.ufrpe.br \\ ${ }^{2}$ Eng $^{\mathrm{a}}$. Agrônoma, Dra ., Unidade Acadêmica de Garanhuns, UFRPE, Garanhuns, PE, Brasil - edilmapg@uag.ufrpe.br \\ ${ }^{3}$ Eng. Agrônomo, Dr., Depto. de Fitotecnia, UFPB, Areia, PB, Brasil - landrade@cca.ufpb.br \\ ${ }^{4}$ Eng. Agrônomo, Depto. de Fitotecnia, UFPB, João Pessoa, PB, Brasil - soareslt@hotmail.com \\ ${ }^{5} \mathrm{Eng}^{\mathrm{a}}$. Agrônoma, Depto. de Fitotecnia, UFPB, João Pessoa, PB, Brasil - ednaagronomia@yahoo.com.br
}

Recebido para publicação: 05/10/2007 - Aceito para publicação: 27/03/2008

\begin{abstract}
Resumo
O trabalho foi desenvolvido na Universidade Federal da Paraíba (CCA/UFPB) com o objetivo de testar diferentes tamanhos de recipientes na produção de mudas de pata-de-vaca (Bauhinia forficata). As sementes foram oriundas de árvores-matrizes localizadas às margens do lago do Departamento de Fitotecnia. Para estudar o comportamento das mudas, empregaram-se sacos plásticos de polietileno com diferentes dimensões: $30 \times 25 \mathrm{~cm}, 30 \times 15 \mathrm{~cm}, 17 \times 15 \mathrm{~cm}$ e $15 \times 9 \mathrm{~cm}$. Para número de folhas, altura das mudas e diâmetro do colo, foi verificado o crescimento linear das mudas ao longo dos períodos de avaliação, em todos os tipos de recipientes, apresentando maiores valores no último período aquelas que estavam no recipiente de 30 × $25 \mathrm{~cm}$. Os recipientes de maior dimensão $(30$ × 25 $\mathrm{cm}$ ) favoreceram maior desenvolvimento vegetativo das mudas de Bauhinia forficata, e os de menor dimensão $(15 \times 9 \mathrm{~cm})$ reduziram a taxa de crescimento das mudas, implicando aumento do ciclo de produção.
\end{abstract}

Palavras-chave: Sacos de polietileno; espécie medicinal; produção de mudas.

\section{Abstract}

Growth of Bauhinia forficata link seedlings in different sizes of recipients. This research was developed at the "Universidade Federal da Paraíba" (CCA/UFPB) with the objective of testing different sizes of recipients in the production of Bauhinia forficata seedlings. The seeds came from seed trees located at the margins of the Department of Fitotecnia lake polyethylene plastic bags were used with different dimensions: $30 \times 25 \mathrm{~cm}, 30 \times 15 \mathrm{~cm}, 17 \times 15 \mathrm{~cm}$ and $15 \times 9 \mathrm{~cm}$. For number of leaves, height of the seedlings and diameter it was detected a linear growth of the seedlings along the evaluation periods, originating from all the recipients, presenting larger values in the last period for those that were been in the recipient $(30 \times 25 \mathrm{~cm})$. The recipients of bigger dimension $(30 \times 25 \mathrm{~cm})$ favored larger vegetative development of the seedlings of Bauhinia forficata and the smallest dimension $(15 \times 9 \mathrm{~cm})$ they reduced the rate of growth of the seedlings, implicating increase of the production cycle.

Keywords: Sacks of polyethylene; medicinal species; production of seedlings.

\section{INTRODUÇ̃̃O}

A essência florestal Bauhinia forficata Link apresenta várias dominações regionais, sendo as mais conhecidas: unha-de-vaca, pata-de-vaca, unha-de-boi e mororó (CORREA, 1984). São plantas semidecíduas, heliófilas, indiferentes quanto às condições de umidade do solo, pioneiras, com características exclusivas da mata semidecídua de altitude, onde ocorre em freqüência elevada, porém de dispersão bastante irregular e descontínua.

A planta é conhecida como medicinal, pois tem ação diurética, antidiabética, tônica renal, depurativa e hipoglicemiante, sendo também utilizada na arborização urbana. Sua madeira é moderadamente pesada, de textura grossa e pouco durável, podendo ser indicada em construção civil, 
para cabo de ferramentas e instrumentos agrícolas, para arcos e raios de carroças, bem como para lenha e carvão (LORENZI, 1998).

A qualidade das mudas é fator fundamental para o sucesso de povoamentos florestais, motivo pelo qual busca-se produzir mudas em grande quantidade e com qualidade. Vários fatores afetam a produção das mudas em viveiro. Entre eles, o tamanho dos recipientes utilizados têm influência direta no custo final da muda, na quantidade de substrato a ser utilizado, no espaço que irá ocupar no viveiro, na mão-de-obra, no transporte, na retirada para a entrega ao produtor, além da influência na quantidade de insumos utilizados (GONZALES et al., 1988).

O tamanho do recipiente ideal para a produção de mudas dependerá do ritmo de crescimento das plantas, o qual é função da espécie e das condições de clima e substrato. Os recipientes mais usados têm capacidade para 40 a $250 \mathrm{~cm}^{3}$, ressaltando-se que as mudas de folhosas tropicais tendem a requerer recipientes maiores do que Pinus e Eucalyptus (EVANS, 1992). No Brasil, para estes dois gêneros, os viveiristas têm utilizado os tubetes de polipropileno de $50 \mathrm{~cm}^{3}$ de capacidade (GONÇALVES; POGGIANI, 1996).

Observa-se, na literatura, que as mudas de diferentes espécies cultivadas em recipientes de maior dimensão possuem maior qualidade. Como exemplo, citam-se trabalhos com Mimosa scabrella em que as mudas cultivadas em recipientes de maior volume apresentaram maior diâmetro de colo e peso de matéria seca da parte aérea e do sistema radicular em relação àquelas cultivadas em recipientes menores (STURION, 1981).

Pereira; Pereira (1985), estudando o tamanho de recipiente para a produção de mudas de seringueira (Hevea brasiliensis Muell. Arg.), verificaram que recipientes de 35 x $15 \mathrm{~cm}$ ou 40 x $15 \mathrm{~cm}$ são tão eficientes quanto os normalmente utilizados $(56 \times 25 \mathrm{~cm})$, apresentando a vantagem adicional de reduzirem os custos de produção, transporte e plantio em cerca de $60 \%$.

Carvalho Filho et al. (2004), estudando a produção de mudas de angelim (Andira fraxinifolia Benth.) em diferentes ambientes, recipientes e substratos, verificaram que o tamanho do recipiente influenciou positivamente o número de folhas.

Cunha et al. (2005) observaram que recipientes de maiores volumes oferecem melhores condições para o desenvolvimento das mudas, mas eles somente devem ser utilizados para espécies que apresentam desenvolvimento lento, necessitando permanecer no viveiro por um longo tempo, ou quando se desejam mudas bem desenvolvidas, para plantio em vias públicas, por exemplo.

De acordo com Sturion; Antunes (2000), a relação altura/diâmetro do colo constitui um dos parâmetros usados para se avaliar a qualidade de mudas florestais, pois, além de refletir o acúmulo de reservas, assegura maior resistência e melhor fixação no solo. Mudas com baixo diâmetro do colo apresentam dificuldades de se manterem eretas após o plantio. Essa variável é reconhecida como um dos melhores, se não o melhor indicador do padrão de qualidade de mudas (MOREIRA; MOREIRA, 1996), sendo, em geral, a mais indicada para determinar a capacidade de sobrevivência de mudas no campo (DANIEL et al., 1997). Dessa forma, mudas com diâmetro do colo pequeno e alturas elevadas são consideradas de qualidade inferior às menores e com maior diâmetro do colo.

O diâmetro e altura dos recipientes podem variar com as características de cada espécie e o respectivo tempo de permanência no viveiro. Gomes et al. (1990) relatam a importância de se estudarem as dimensões dos recipientes para a produção das mudas de ipê (Tabebuia serratifolia (Vahl) Nich.), copaíba (Copaifera langsdorffii Desf.) e angico-vermelho (Piptadenia peregrina (L.) Benth.). Apesar de a literatura já relatar várias pesquisas com esse objetivo, trabalhos dessa natureza são considerados importantes, pois o uso de recipientes maiores que os recomendáveis resulta em gastos desnecessários com substratos, aumentando os custos ainda no viveiro.

Diante do exposto, o presente trabalho teve como objetivo testar diferentes tamanhos de recipientes na produção de mudas de pata-de-vaca.

\section{MATERIAL E MÉTODOS}

O trabalho foi desenvolvido no viveiro do Departamento de Fitotecnia da Universidade Federal da Paraíba (CCA/UFPB), em Areia (PB). As sementes de pata-de-vaca (Bauhinia forficata Link.) foram oriundas de árvores-matrizes localizadas às margens do lago do Departamento de Fitotecnia, e, para se estudar o comportamento das mudas de pata-de-vaca, empregaram-se sacos plásticos de polietileno com 
diferentes dimensões: 1 - saco plástico de $30 \times 25 \mathrm{~cm}$; 2- saco plástico de $30 \times 15 \mathrm{~cm}$; 3- saco plástico de 17 x $15 \mathrm{~cm}$; e 4- saco plástico de $15 \times 9 \mathrm{~cm}$.

Previamente à instalação, as sementes foram submetidas ao teste de germinação, para avaliação da qualidade fisiológica, empregando-se quatro bandejas marmitex com areia lavada e com quatro repetições de 25 sementes.

Os sacos plásticos foram preenchidos com terra vegetal devidamente peneirada, com composição química identificada na tabela 1. Foram semeadas em cada saco três sementes, e após três semanas foram realizados desbastes das plântulas em excesso, permanecendo apenas uma por recipiente.

Tabela 1. Análise química da terra vegetal utilizada na produção de mudas de Bauhinia forficata.

Table 1. Chemical analysis of the vegetable composition used in the production of seedlings of the Bauhinia forficata.

\begin{tabular}{lc}
\hline Característica química* & Valor \\
\hline M.O. $(\mathrm{g} / \mathrm{kg})$ & 7,18 \\
$\mathrm{P}\left(\mathrm{mg} / \mathrm{dm}^{3}\right)$ & 59,68 \\
$\mathrm{~K}\left(\mathrm{mg} / \mathrm{dm}^{3}\right)$ & 67,1 \\
$\mathrm{Ca}\left(\mathrm{cmol}_{\mathrm{c}} / \mathrm{dm}^{3}\right)$ & 3,4 \\
$\mathrm{Mg}\left(\mathrm{cmol}_{\mathrm{c}} / \mathrm{dm}^{3}\right)$ & 0,5 \\
\hline * Análise de fertilidade realizada pelo Laboratório de Química e Fertilidade do Solo do CCA/UFPB.
\end{tabular}

A umidade dos substratos foi mantida com regas periódicas, sempre que necessário, realizandose capinas no solo e dentro dos sacos.

As mudas ficaram no viveiro por um período de 72 dias após a semeadura, sendo a primeira avaliação feita aos vinte e um dias, perfazendo oito períodos de avaliações. Para avaliação do crescimento inicial das mudas, mediram-se:

- Número de folhas: avaliou-se o número de folhas emitidas semanalmente e o número de brotos.

- Altura de plântulas: com o auxílio de uma trena, procedeu-se à medição da altura das plantas de patade-vaca, medindo-se a distância entre o ápice da planta e o colo.

- Diâmetro das plântulas: foi obtido medindo-se a secção do colo das mudas com o auxílio de um paquímetro.

Ao término das avaliações na parte aérea das plântulas, as mudas foram retiradas dos sacos e, com o auxílio de uma mangueira com água corrente e uma peneira, foram retirados os substratos, avaliando-se:

- Comprimento da raiz primária: com auxílio de uma régua, mediram-se as raízes de todas as plântulas (os resultados foram expressos em cm por plântulas).

- Massa seca da parte aérea e da raiz: a parte aérea e da raiz das plântulas foram separadas com a ajuda de um estilete e levadas separadamente dentro de sacos de papel para a estufa, a uma temperatura de $65^{\circ} \mathrm{C}$, até a obtenção do peso constante, sendo posteriormente pesadas em balança eletrônica.

$\mathrm{O}$ delineamento estatístico utilizado foi o inteiramente ao acaso, com quatro tratamentos e oito períodos de avaliação e quatro repetições de 10 recipientes. As médias foram comparadas pelo Teste de Tukey, em nível de 5\% de probabilidade, e os períodos foram avaliados por regressão polinomial, com auxílio do programa de estatística SAEG 5.0.

\section{RESULTADOS E DISCUSSÃO}

Inicialmente, as sementes de pata-de-vaca apresentaram um percentual de germinação de 52\%, justificando-se a semeadura de três sementes por recipiente.

O número médio de folhas por plântulas apresentou uma interação significativa entre os recipientes testados (Tabela 2). De acordo com os resultados, contatou-se que houve maior influência do recipiente $1(30 \times 25 \mathrm{~cm})$ sobre as mudas a partir do quinto período, sendo mais evidenciada no sétimo e oitavo períodos de avaliação. Os menores números de folhas foram observados no sétimo e oitavo período nas mudas do recipiente $4(15 \times 9 \mathrm{~cm})$. Isso sugere que, provavelmente, o tamanho do recipiente 
tenha limitado não somente o volume, mas a quantidade de nutrientes disponíveis para o sistema radicular, afetando a distribuição para parte aérea, o que refletiu no número de folhas por plântulas.

Tabela 2. Número de folhas de mudas de pata-de-vaca provenientes de diferentes recipientes e períodos de avaliação.

Table 2. Number of leaves of seedlings of the Bauhinia forficata coming from different recipients and evaluation periods.

\begin{tabular}{lcccccccc}
\hline \multirow{2}{*}{ Recipientes } & \multicolumn{7}{c}{ Períodos (semanas) } \\
\cline { 2 - 8 } & $\mathbf{1}^{\mathbf{0}}$ & $\mathbf{2}^{\mathbf{0}}$ & $\mathbf{3}^{\mathbf{0}}$ & $\mathbf{4}^{\mathbf{0}}$ & $\mathbf{5}^{\mathbf{0}}$ & $\mathbf{6}^{\mathbf{0}}$ & $\mathbf{7}^{\mathbf{0}}$ & $\mathbf{8}^{\mathbf{0}}$ \\
\hline $1(30 \times 25 \mathrm{~cm})$ & $2,0 \mathrm{~A}$ & $2,9 \mathrm{~A}$ & $3,7 \mathrm{~A}$ & $4,7 \mathrm{~A}$ & $6 \mathrm{~A}$ & $7 \mathrm{~A}$ & $9,2 \mathrm{~A}$ & $10,8 \mathrm{~A}$ \\
$2(30 \times 15 \mathrm{~cm})$ & $1,8 \mathrm{~A}$ & $2,8 \mathrm{~A}$ & $3,4 \mathrm{~A}$ & $4,4 \mathrm{~A}$ & $5,5 \mathrm{AB}$ & $6,9 \mathrm{~B}$ & $8,1 \mathrm{~B}$ & $9,1 \mathrm{~B}$ \\
$3(17 \times 15 \mathrm{~cm})$ & $1,9 \mathrm{~A}$ & $2,9 \mathrm{~A}$ & $3,5 \mathrm{~A}$ & $4,8 \mathrm{~A}$ & $5,5 \mathrm{AB}$ & $6,1 \mathrm{AB}$ & $6,3 \mathrm{C}$ & $6,8 \mathrm{C}$ \\
$4(15 \times 9 \mathrm{~cm})$ & $1,7 \mathrm{~A}$ & $2,9 \mathrm{~A}$ & $3,2 \mathrm{~A}$ & $4,1 \mathrm{~A}$ & $4,7 \mathrm{~B}$ & $4 \mathrm{~B}$ & $5,6 \mathrm{C}$ & $6,1 \mathrm{C}$ \\
\hline
\end{tabular}

Letras maiúsculas comparam os recipientes em cada período de avaliação.

A figura 1 apresenta o comportamento das plântulas quanto à emissão de folhas nos diferentes recipientes durante oito períodos de avaliação. Dos recipientes estudados, aquele de maior dimensão proporcionou maior desenvolvimento da folha, durante os períodos de avaliação. Carvalho Filho et al. (2004), estudando a produção de mudas de angelim (Andira fraxinifolia Benth.) em diferentes ambientes, recipientes e substratos, também verificaram que o tamanho do recipiente influenciou positivamente o número de folhas.

Recipiente $1 \quad$ Recipiente 2

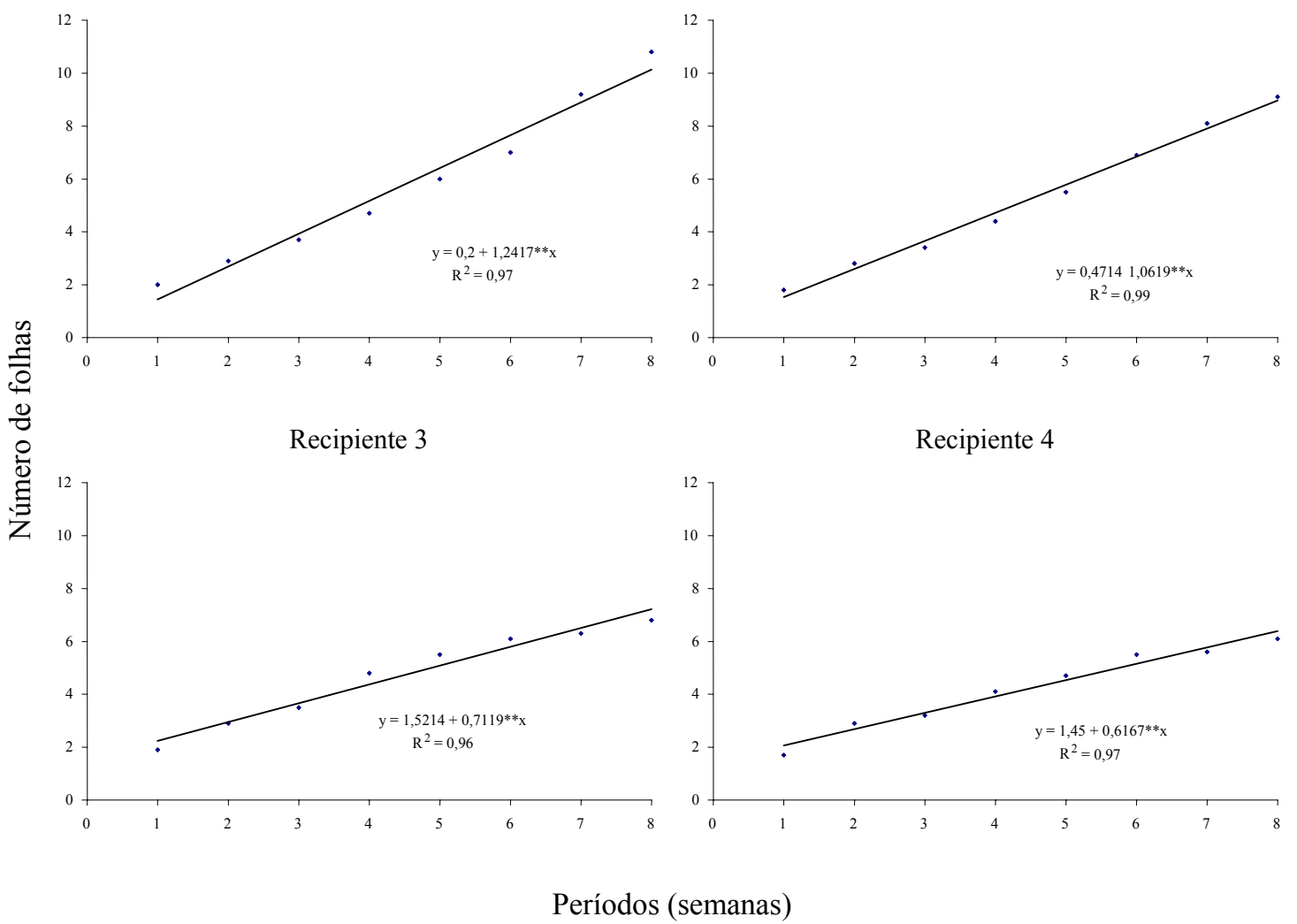

Figura 1. Número de folhas de mudas de pata-de-vaca em diferentes tamanhos de recipientes e períodos de avaliação.

Figure 1. Number of leaves of Bauhinia forficata seedlings in different sizes of recipients and evaluation periods. 
Quando as plântulas foram avaliadas quanto à sua altura (Tabela 3), constatou-se que o uso de sacos de maior dimensão (recipiente 1) proporcionou maior média de altura da parte aérea já a partir do quinto período. Resultados similares foram encontrados com outras espécies, tais como os observados em mudas de camu-camu (Myrciaria dubia L.) produzidas em sacos de polietileno preto de $21 \times 19 \mathrm{~cm}$, que mostraram tendência de melhor desenvolvimento quando comparadas com outras cultivadas em recipientes menores (YUYAMA; SIQUEIRA, 1999). Da mesma forma, Oliveira et al. (2000) observaram que mudas de cajueiro propagadas em sacos de polietileno apresentaram altura estatisticamente superior a mudas da mesma espécie propagadas em tubetes, com volumes inferiores de substratos que os dos sacos.

Tabela 3. Altura $(\mathrm{cm})$ da parte aérea das mudas de Bauhinia forficata obtidas de diferentes recipientes e períodos de avaliação.

Table 3. Height (cm) of the aerial part of Bauhinia forficata seedlings obtained from different recipients and evaluation periods.

\begin{tabular}{lcccccccc}
\hline \multirow{2}{*}{ Recipientes } & \multicolumn{9}{c}{ Períodos (semanas) } \\
\cline { 2 - 8 } & $\mathbf{1}^{\mathbf{0}}$ & $\mathbf{2}^{\mathbf{0}}$ & $\mathbf{3}^{\mathbf{0}}$ & $\mathbf{4}^{\mathbf{0}}$ & $\mathbf{5}^{\mathbf{0}}$ & $\mathbf{6}^{\mathbf{0}}$ & $\mathbf{7}^{\mathbf{0}}$ & $\mathbf{8}^{\mathbf{0}}$ \\
\hline $1(30 \times 25 \mathrm{~cm})$ & $11,75 \mathrm{~A}$ & $13,22 \mathrm{~A}$ & $16,77 \mathrm{~A}$ & $20,17 \mathrm{~A}$ & $23,48 \mathrm{~A}$ & $29,15 \mathrm{~A}$ & $33,75 \mathrm{~A}$ & $39,12 \mathrm{~A}$ \\
$2(30 \times 15 \mathrm{~cm})$ & $11,13 \mathrm{~A}$ & $12,75 \mathrm{~A}$ & $16,17 \mathrm{~A}$ & $18,93 \mathrm{~A}$ & $21,05 \mathrm{AB}$ & $25,9 \mathrm{~B}$ & $27,81 \mathrm{~B}$ & $30,35 \mathrm{~B}$ \\
$3(17 \times 15 \mathrm{~cm})$ & $11,23 \mathrm{~A}$ & $12,87 \mathrm{~A}$ & $17,59 \mathrm{~A}$ & $20,28 \mathrm{~A}$ & $22,44 \mathrm{AB}$ & $23,91 \mathrm{BC}$ & $25,35 \mathrm{BC}$ & $25,25 \mathrm{C}$ \\
$4(15 \times 9 \mathrm{~cm})$ & $11,26 \mathrm{~A}$ & $13,39 \mathrm{~A}$ & $16,56 \mathrm{~A}$ & $19,12 \mathrm{~A}$ & $19,91 \mathrm{~B}$ & $21,53 \mathrm{C}$ & $22,25 \mathrm{C}$ & $22,41 \mathrm{C}$ \\
\hline
\end{tabular}

Letras maiúsculas comparam os recipientes em cada período de avaliação.

O comportamento da altura das mudas de pata-de-vaca provenientes de diferentes tamanhos de recipientes pode ser observado na figura 2. Foi verificado um crescimento linear ao longo dos períodos de avaliação das mudas oriundas de todos os recipientes, com as maiores médias obtidas no último período para o recipiente $1(30 \times 25 \mathrm{~cm})$. Mais uma vez, fica evidente a influência positiva do tamanho do recipiente no crescimento das mudas. Cunha et al. (2005), estudando os efeitos de substratos e das dimensões dos recipientes na qualidade das mudas de Tabebuia impetiginosa (Mart. ex D.C.) Standl., verificaram que elas podem ser satisfatoriamente produzidas em sacos de polietileno de $32 \times 15 \mathrm{~cm}$, utilizando o substrato terra de subsolo + composto orgânico, produzido com material de baixo custo, geralmente disponível no próprio meio rural.

Para a avaliação do diâmetro do colo das mudas de pata-de-vaca (Tabela 4), as maiores médias foram verificadas, depois de decorridas sete semanas, nas mudas oriundas do recipiente 1, diferindo estatisticamente daquelas conduzidas em sacos de dimensão inferior. O efeito positivo do tamanho do recipiente sobre o crescimento e desenvolvimento das mudas já foi verificado quando estudadas outras variáveis neste trabalho. $\mathrm{O}$ tamanho de recipiente ideal para a produção de mudas dependerá do ritmo de crescimento das plantas, o qual é função da espécie e das condições de clima e substrato. Pereira; Pereira (1985), estudando o tamanho de recipiente para a produção de mudas de seringueira (Hevea brasiliensis Muell. Arg.), encontraram que recipientes de $35 \times 15 \mathrm{~cm}$ ou de $40 \times 15 \mathrm{~cm}$ são tão eficientes quanto os normalmente utilizados (56 x $25 \mathrm{~cm}$.), apresentando a vantagem adicional de reduzirem os custos de produção, transporte e plantio em cerca de $60 \%$.

A maior ascendência é novamente verificada para aqueles caules oriundos de mudas cultivadas com maior volume de substrato, proporcionado pelo tratamento 1. Segundo Carvalho Filho et al. (2004), para a produção de mudas de angelim deve ser usado um substrato contendo solo + esterco (2:1) e/ou solo + areia + esterco $(1: 2: 1)$ em sacos de polietileno $20 \times 15 \mathrm{~cm}$.

Daniel et al. (1997) relataram que, em geral, o diâmetro do colo é analisado para indicar a capacidade de sobrevivência da muda no campo, além de ser parâmetro para a definição de doses de fertilizantes a serem aplicadas na produção de mudas.

Mudas com baixo diâmetro do colo apresentam dificuldades de se manterem eretas após o plantio. Essa variável é reconhecida como um dos melhores, se não o melhor indicador do padrão de qualidade de mudas (MOREIRA; MOREIRA, 1996), sendo, em geral, o mais indicado para determinar a capacidade de sobrevivência de mudas no campo (DANIEL et al., 1997). Dessa forma, mudas com diâmetro do colo pequeno e alturas elevadas são consideradas de qualidade inferior às menores e com maior diâmetro do colo. 


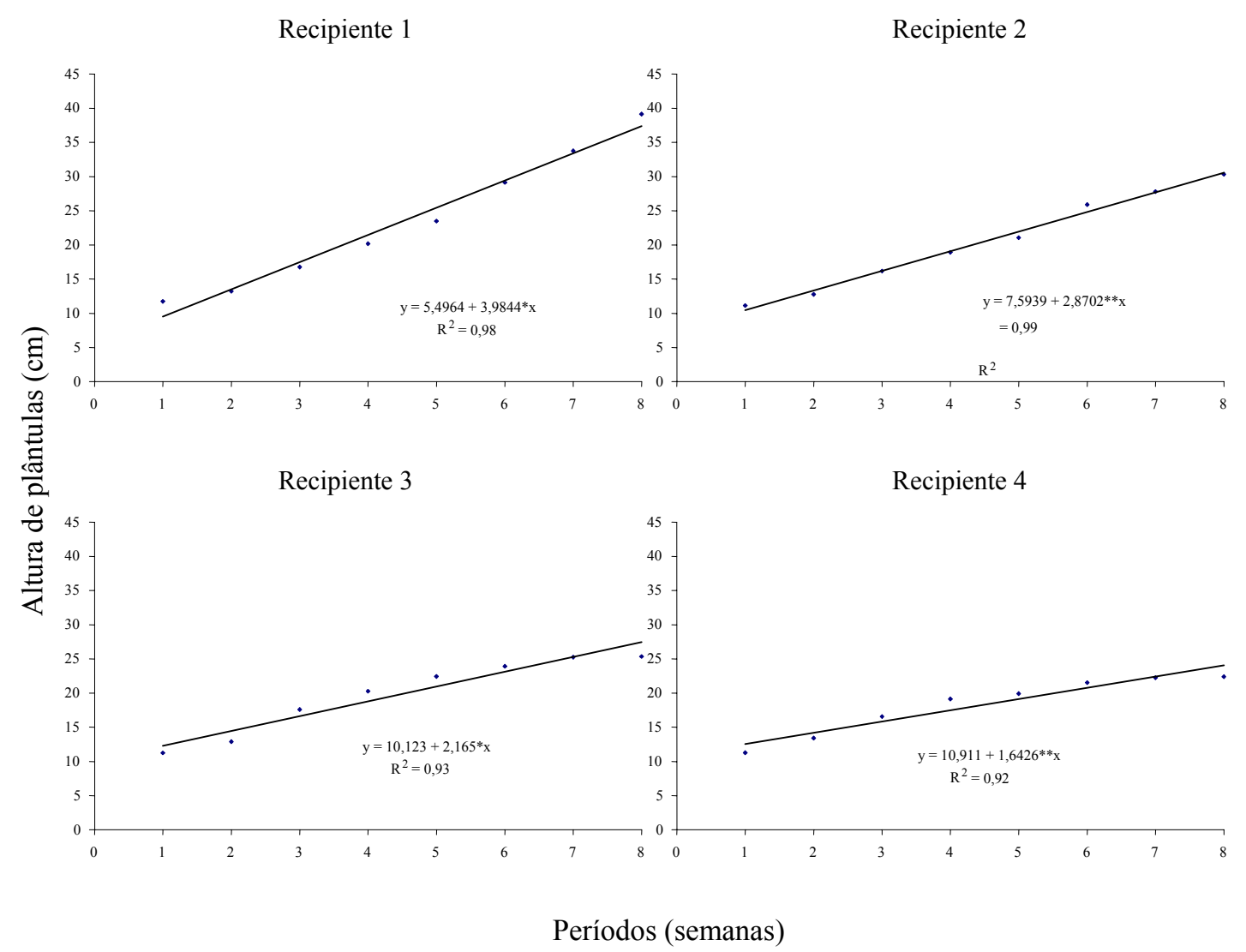

Figura 2. Altura $(\mathrm{cm})$ das mudas de Bauhinia forficata durante oito períodos de avaliação.

Figure 2. Height $(\mathrm{cm})$ of Bauhinia forficata seedlings during eight evaluation periods.

Tabela 4. Diâmetro (cm) do colo das mudas de Bauhinia forficata obtidas de diferentes recipientes e períodos de avaliação.

Table 4. Stem diameter $(\mathrm{cm})$ of the Bauhinia forficate seedlings obtained from different recipients and evaluation periods.

\begin{tabular}{lrrrrrrrr}
\hline \multirow{2}{*}{ Recipientes } & \multicolumn{7}{c}{ Períodos (semanas) } \\
\cline { 2 - 8 } & $\mathbf{1}^{\mathbf{0}}$ & $\mathbf{2}^{\mathbf{0}}$ & $\mathbf{3}^{\mathbf{0}}$ & $\mathbf{4}^{\mathbf{0}}$ & \multicolumn{1}{c}{$\mathbf{5}^{\mathbf{0}}$} & $\mathbf{6}^{\mathbf{0}}$ & $\mathbf{7}^{\mathbf{0}}$ & $\mathbf{8}^{\mathbf{0}}$ \\
\hline $1(30 \times 25 \mathrm{~cm})$ & $0,24 \mathrm{~A}$ & $0,26 \mathrm{~A}$ & $0,28 \mathrm{~A}$ & $0,30 \mathrm{~A}$ & $0,36 \mathrm{~A}$ & $0,44 \mathrm{~A}$ & $0,49 \mathrm{~A}$ & $0,57 \mathrm{~A}$ \\
$2(30 \times 15 \mathrm{~cm})$ & $0,23 \mathrm{~A}$ & $0,25 \mathrm{~A}$ & $0,27 \mathrm{~A}$ & $0,30 \mathrm{~A}$ & $0,34 \mathrm{AB}$ & $0,40 \mathrm{AB}$ & $0,44 \mathrm{~B}$ & $0,47 \mathrm{~B}$ \\
$3(17 \times 15 \mathrm{~cm})$ & $0,27 \mathrm{~A}$ & $0,24 \mathrm{~A}$ & $0,28 \mathrm{~A}$ & $0,30 \mathrm{~A}$ & $0,33 \mathrm{AB}$ & $0,38 \mathrm{~B}$ & $0,41 \mathrm{BC}$ & $0,44 \mathrm{~B}$ \\
$4(15 \times 9 \mathrm{~cm})$ & $0,26 \mathrm{~A}$ & $0,24 \mathrm{~A}$ & $0,26 \mathrm{~A}$ & $0,28 \mathrm{~A}$ & $0,30 \mathrm{~B}$ & $0,35 \mathrm{C}$ & $0,37 \mathrm{C}$ & $0,39 \mathrm{C}$ \\
\hline
\end{tabular}

Letras maiúsculas comparam os recipientes em cada período de avaliação.

Neste estudo, as variáveis estudadas responderam positivamente aos tamanhos dos recipientes, ou seja, quanto maior o volume do recipiente, maior o resultado para todas as variáveis estudadas. O diâmetro e a altura dos recipientes podem variar com as características de cada espécie e com o respectivo tempo de permanência no viveiro. Gomes et al. (1990) relatam a importância de se estudarem as dimensões dos recipientes para a produção das mudas, uma vez que o uso de recipientes maiores que os recomendáveis resulta em custos desnecessários de substratos para a produção de mudas de ipê (Tabebuia serratifolia (Vahl) Nich.), copaíba (Copaifera langsdorffii Desf.) e angico-vermelho (Piptadenia peregrina (L.) Benth.). 

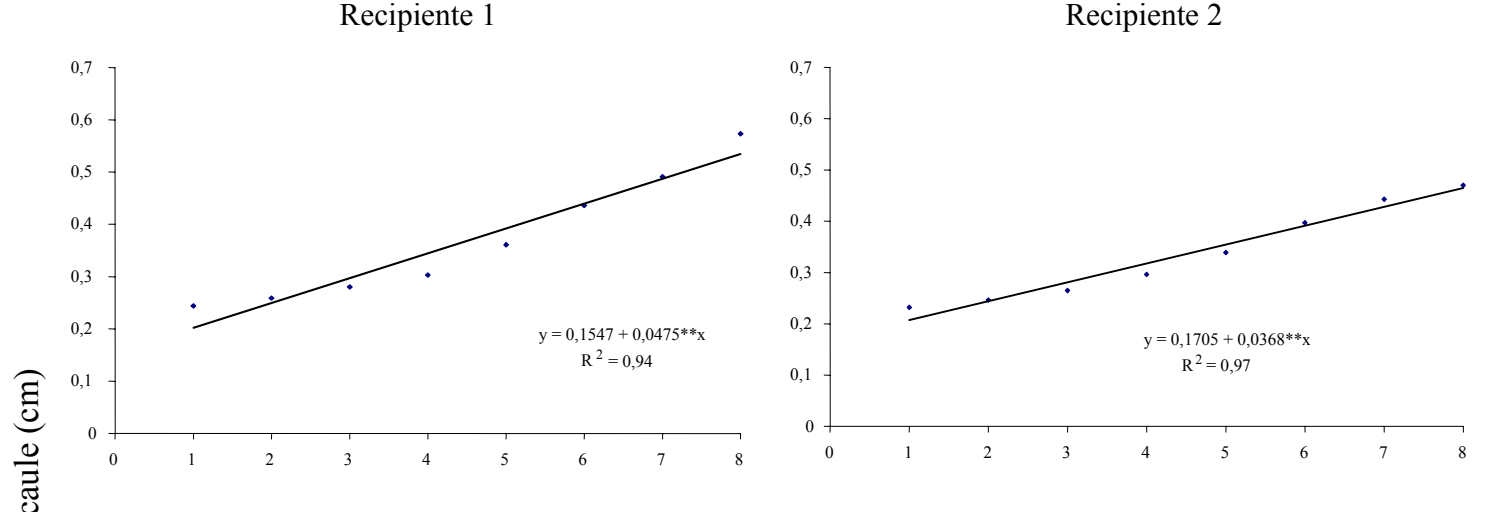

\section{Recipiente 3}

Recipiente 4

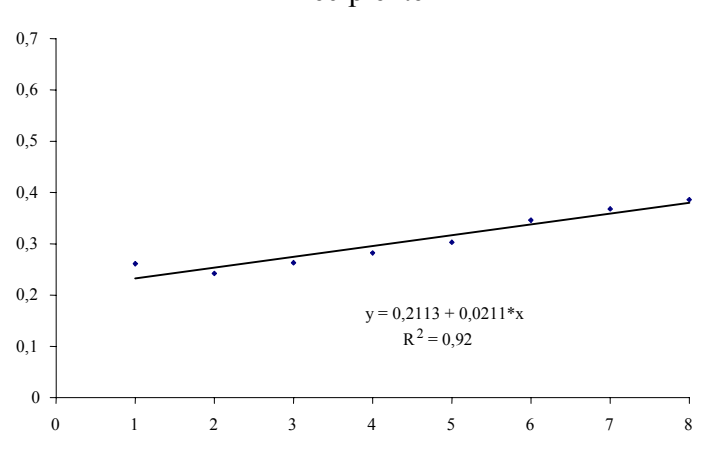

Períodos (semanas)

Figura 3. Diâmetro $(\mathrm{cm})$ do colo de plântulas de Bauhinia forficata em diferentes recipientes e períodos de avaliação.

Figure 3. Diameter $(\mathrm{cm})$ of the colo of Bauhinia forficata seedlings from different recipients and evaluation periods

Na tabela 5 encontram-se os resultados de comprimento da raiz primária e da massa seca da parte aérea e da raiz das mudas de pata-de-vaca. O crescimento e a massa seca das mudas de pata de vaca estão diretamente relacionados com o tamanho dos recipientes. O recipiente com maior volume de substrato proporcionou os maiores valores e o menor resultado foi encontrado quando as mudas estavam no menor recipiente. Gomes et al. (1980, 1990), trabalhando com mudas de Pinus caribaea var. hondurensis, Tabebuia serratifolia, Copaifera longsdorffi e Piptadenia sp, também concluíram que existe uma relação direta entre o tamanho do recipiente e o ganho em massa seca das mudas.

Tabela 5. Comprimento da raiz (cm), massa seca da parte aérea $(\mathrm{g})$ e raiz $(\mathrm{g})$ das mudas de Bauhinia forficata provenientes de diferentes recipientes.

Table 5. Length of the root, dry mass of the aerial part and root of the seedlings of Bauhinia forficate coming from different recipients.

\begin{tabular}{lccc}
\hline Recipientes & Comprimento da raiz & Massa seca da parte aérea & Massa seca da raiz \\
\hline $1(30 \times 25 \mathrm{~cm})$ & $30,35 \mathrm{~A}$ & $4,3814 \mathrm{~A}$ & $1,4461 \mathrm{~A}$ \\
$2(30 \times 15 \mathrm{~cm})$ & $23,05 \mathrm{~B}$ & $1,9349 \mathrm{~B}$ & $1,0483 \mathrm{~B}$ \\
$3(17 \times 15 \mathrm{~cm})$ & $16,20 \mathrm{C}$ & $1,1278 \mathrm{~B}$ & $0,7370 \mathrm{BC}$ \\
$4(15 \times 9 \mathrm{~cm})$ & $13,20 \mathrm{C}$ & $0,6852 \mathrm{~B}$ & $0,4882 \mathrm{C}$ \\
\hline
\end{tabular}

Letras maiúsculas comparam os recipientes em cada variável. 
Conforme relatam Jesus et al. (1987), as dimensões do recipiente e o tipo de substrato influenciam a qualidade das mudas, e isso se deve, possivelmente, ao fato de esses elementos estarem diretamente relacionados com a disponibilidade de espaço físico e o aporte de nutrientes, condições essenciais para o cultivo de plantas ex situ.

De acordo com Cunha et al. (2005), recipientes de maiores volumes oferecem melhores condições para o desenvolvimento das mudas, contudo eles somente devem ser utilizados para espécies que apresentam desenvolvimento lento, necessitando permanecer no viveiro por um longo tempo, ou quando se desejam mudas bem desenvolvidas, para plantio em vias públicas, por exemplo.

\section{CONCLUSÃO}

As mudas de Bauhinia forficata até 49 dias (quinta semana) podem ser produzidas em recipientes com dimensões de $30 \times 25 \mathrm{~cm}, 30 \times 15 \mathrm{~cm}$ e $17 \times 15 \mathrm{~cm}$, sendo que depois desse período é recomendado o recipiente com $30 \times 25 \mathrm{~cm}$, pois este favorece maior crescimento vegetativo.

Até o quarto período (42 dias) o menor recipiente $(15 \times 9 \mathrm{~cm})$ promoveu taxa de crescimento das mudas semelhante aos recipientes de maiores dimensões; a partir desse período, reduziu a taxa de crescimento das mudas, implicando aumento do ciclo de produção.

\section{REFERÊNCIAS}

CARVALHO FILHO, J. L.; ARRIGONI-BLANK, M. F.; BLANK, F. A. Produção de mudas de angelim (Andira fraxinifolia Benth.) em diferentes ambientes, recipientes e substratos. Ciência Agronômica, Fortaleza, v. 35, n. 1, p. $61-67$, jan./jun. 2004.

CORRÊA, M. P. Dicionário das plantas úteis do Brasil e das exóticas cultivadas. Rio de Janeiro: Ministério da Agricultura - IBDF, 1984. v. 2. p. 324.

CUNHA, A. O.; ANDRADE, L. A.; BRUNO, R. L. A.; SIlVA, J. A. L.; SOUZA, V. C. Efeitos de substratos e das dimensões dos recipientes na qualidade das mudas de Tabebuia impetiginosa (Mart. Ex D.C.) Standl. Revista Árvore, Viçosa, MG, v. 29, n. 4, p. 507-516, 2005.

DANIEL, O.; VITORINO, A. C. T.; ALOISI, A. A.; MAZZOCHIN, L.; TOKURA, A. M.; PINHEIRO, E. R.; SOUZA, E. F. Aplicação de fósforo em mudas de Acacia mangium. Revista Árvore, Viçosa, MG, v. 21, n. 2, p. 163-168, 1997.

EVANS, J. Plantation forestry in the tropics. Oxford: Clarendon, 1992. $403 \mathrm{p}$.

GOMES, J. M.; COUTO, L.; BORGES, R. C. G. FREITAS, S. C. Influência do tamanho da embalagem plástica na produção de mudas de Ipê, Copaiba e Angico Vermelho. Revista Árvore, Viçosa, MG, v. 14, n. 1, p. 26-34, 1990.

GOMES, J. M.; PEREIRA, A. R.; MORAIS, E. J. Influência do tamanho da embalagem na produção de mudas de Pinus caribaea var. hondurensis. Revista Árvore, Viçosa, MG, v. 9, n. 1, p. 16-20, 1980.

GONÇALVES, J. L. M.; POGGIANI, F. Substratos para produção de mudas florestais. In: SOLOSUELO-CONGRESSOLATINO AMERICANO DE CIÊNCIA DO SOLO, 1996, Águas de Lindóia-SP. Resumos expandidos... Águas de Lindóia: SLCS; SBCS; ESALQ/USP; CEA-ESALQ/USP; SBM, 1996. 1 CD Rom.

GONZALES, R. A.; PEREZ, S. M.; BLANCO, J. J. Estudio sobre el comportamiento en vivero de Pinus caribaea var. Caribaea cultivado en envases de polietileno de 12 dimensiones diferentes. Forestal Baracoa, Cuba, v. 18, n. 1, p. 39-51, 1988.

JESUS, R. M. de; MENANDRO, M. de S. Efeito do tamanho do recipiente, tipo de substrato e sombreamento na produção de mudas de louro (Cordia trichotoma (Vell.) Arrab.) e gonçalo-alves (Astronium fraxinifolium Schott). IPEF, Piracicaba, n. 37, p. 13-19, 1987.

LORENZI, H. Árvores brasileiras: manual para identificação e cultivo de plantas arbóreas do Brasil. Nova Odessa: Plantarum, 1998. 352 p. 
MOREIRA, F. M. S.; MOREIRA, F. W. Característica de germinação de 64 espécies de leguminosas florestais nativas da Amazônia, em condições de viveiro. Acta Amazônica, Manuas, v. 26, n. 1/2, p. 316, 1996.

OLIVEIRA, V. H.; LIMA, R. N.; PINHEIRO, R. D. Efeito do recipiente utilizado na formação de mudas no crescimento e desenvolvimento de plantas de cajueiro cultivadas sob irrigação. Fortaleza: Embrapa Agroindústria Tropical, 2000. 3 p. (Pesquisa em andamento, n. 72)

PEREIRA, A. V.; PEREIRA, E. B. C. Influência do tamanho do saco plástico no desenvolvimento de mudas de seringueira, durante a fase de viveiro. EMBRAPA/CNPSD, Manaus, v. 38, p. 1-7, 1985.

STURION, J. A. Produção de mudas Mimosa scabella Benth. In: SEMINÁRIO SOBRE ATUALIDADES E PERSPECTIVAS FLORESTAIS. "Bracatinga uma alternativa para reflorestamento", 4., 1981, Curitiba. Anais... Curitiba: EMBRAPA/URPFCS, 1981. (EMBRAPA-URPFCS Documentos, n. 5).

STURION, J. A.; ANTUNES, B. M. A. Produção de mudas de espécies florestais. In: GALVÃO, A .P. M. Reflorestamento de propriedades rurais para fins de produtivos e ambientais. Colombo: EMBRAPA Florestas, 2000. p. 125-150.

YUYAMA, K.; SIQUEIRA, J. A. S. Efeito do tamanho das sementes e do recipiente no crescimento de mudas de camu-camu (Myrciaira dubia). Acta Amazônica, Manaus, v. 29, n. 4, p. 647- 650, 1999. 